\title{
The Multiplicity of Solutions for a Certain Class of Fractional Elliptic Equation
}

\author{
Shifeng Zhang*, Zhiyang Jia and Jihe Wang \\ Tourism and Culture College of Yunnan University, Lijiang, China \\ *Corresponding author
}

\begin{abstract}
This paper explores the multiplicity of solutions for a Certain class of fractional elliptic equation under the Dirichlet boundary conditions. By using the asymptotic property of the nonlinear term $f(x, u)$ at zero and at infinite point, the mountain pass theorem and proper truncation methods can be applied to get both a positive and a negative solution for all parameters under the condition of not satisfying the Ambrosetti-Rabinowitz.
\end{abstract}

Keywords-fractional laplace's equation; dirichlet boundary value condition; mountain pass theorem; positive solution; negative solution.

\section{INTRODUCTION}

In this paper, we study the multiplicity of solutions for the following fractional elliptic:

$$
\left\{\begin{array}{r}
(-\Delta)^{s} u=\lambda a(x)|u|^{r-2} u+\lambda f(x, u) ; x \in \Omega \\
u=0 ; x \in \partial \Omega
\end{array}\right.
$$

Where $\Omega \in R^{N}(N \geq 2)$ is a bounded domain with smooth boundary $\partial \Omega \cdot(-\Delta)^{S}$ is the Fractional Laplace operator, $s \in(0,1), \quad f \in C(\bar{\Omega} \times \mathrm{R}, \mathrm{R})$.

Fractional Laplace operator $(-\Delta)^{s}$ is an infinitesimal

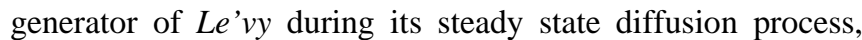
which has been widely used in many fields. In order to study the nontrivial solution of nonlinear equations, AR condition is often used to ensure the compactness of corresponding energy. In recent years, many researchers have confronted with the multiplicity of various nonlinear solutions when it fails to satisfy the AR condition, but researches of multiplicity the fractional order elliptic equations has gradually got more attention in recent years . In this paper, we mainly explore the multiplicity of solutions of fractional order elliptic equations with parameters. With mountain pass theorem, we get both a positive and a negative solution for all parameters while not satisfying the Ambrosetti-Rabinowitz.

Note:

$$
H(x, \mathrm{u})=\mathrm{u} f(x, u)-2 F(x, u) \text { and } F(x, u)=\int_{0}^{u} f(x, t) d t .
$$

Suppose the function $f(x, u)$ on (1) meet the following conditions:
(H) $\lim _{|t| \rightarrow 0} \frac{f(x, t)}{t}=0$ uniformly for almost all $x \in \Omega$;

$\left(\boldsymbol{H}_{2}\right)$ there exists positive $C_{1}, C_{2}, p$, and:

$$
\begin{gathered}
|f(x, u)| \leq C_{1}+C_{2}|t|^{p} \\
1<p<\frac{N+2 s}{N-2 s}, \forall t \in R, \text { a.e. } x \in \Omega
\end{gathered}
$$

(H) $\lim _{|t| \rightarrow+\infty} \frac{f(x, t)}{t^{2}}=+\infty$ uniformly for a.a $x \in \Omega$;

$\left(\boldsymbol{H}_{4}\right)$ there exists $\mathrm{C}^{*}>0$ such that $\mathrm{H}\left(x, t_{1}\right) \leq \mathrm{H}\left(x, t_{2}\right)+\mathrm{C}^{*}$ for all $0<t_{1}<t_{2}$ or $t_{2}<t_{1}<0$ and uniformly for a.e. $x \in \Omega$.

\section{MAIN RESULTS}

Theorem 1. If hypotheses $\left(H_{1}\right)-\left(H_{4}\right)$ is available, then Problem (1) have at least a positive solution and a negative answer when $\lambda>0$.

Notes 1: in [1], the multiplicity of solutions of $(-\Delta)^{s} u=f(x, u)$ is obtained under the condition of satisfying the Ambrosetti-Rabinowitz.

It is the multiplicity of solutions of $-\Delta_{p}(b(x) u)=a(x)|u|^{r-2} u+f(x, u)$ in [2]. When the (AR) condition is not satisfied, [3] discussed the multiplicity of solutions for problem (1) when $s=1$. In this paper, under the influence of the parameters $\lambda$, we discussed the multiplicity of solutions for fractional Laplacian equations (1) when the (AR) condition is not satisfied.

\section{BACKGROUND KNOWLEDGE}

We can denote $\left\{\varphi_{k}\right\}$ as A set of orthogonal basis of $L_{2}(\Omega)$, with $\left\|\varphi_{k}\right\|_{2}=1$, and make up the spectrum decomposition of $(-\mathrm{V})^{s}$ under the Dirichlet boundary condition. $\lambda_{k}$ is the corresponding characteristic value .

Define:

$$
H_{0}^{s}(\Omega)=\left\{u=\sum_{k=0}^{\infty} a_{k} \varphi_{k} \in L^{2}(\Omega):\|u\|_{H_{0}^{s}(\Omega)}=\left(\sum_{k=0}^{\infty} a_{k}{ }^{2} \varphi_{k}^{s}\right)^{\frac{1}{2}}<\infty\right\} .
$$


Denote $H^{-s}(\Omega)$ as dual space of $H_{0}^{s}(\Omega), u \in H_{0}^{s}(\Omega)$, $u=\sum_{k=0}^{\infty} a_{k} \varphi_{k}$ with $a_{k}=\int_{\Omega} u \varphi_{k} d x$.We define $(-\mathrm{V})^{s}$ as: $(-V)^{s} u=\sum_{k=0}^{\infty} a_{k} \lambda_{k}^{s} \varphi_{k} \in H^{-s}(\Omega)$.

$\left\{\left(\varphi_{k}, \lambda_{k}{ }^{s}\right)\right\}$ is the characteristic function and the characteristic value of $(-\mathrm{V})^{s}$.

Define inner product in $H_{0}^{s}(\Omega)$ as:

$$
(u, v)_{H_{0}^{s}(\Omega)}=\int_{\Omega}(-\mathrm{V})^{\frac{s}{2}} u(-\mathrm{V})^{\frac{s}{2}} v d x .
$$

It is easy to find that $H_{0}^{s}(\Omega)$ is a Hilbert space.

Define $u \in H_{0}^{s}(\Omega) \quad$ is a weak solution on (1), if $\int_{\Omega}(-V)^{\frac{s}{2}} u(-V)^{\frac{s}{2}} v d x=\lambda \int_{\Omega} a(x)|u|^{r-2} u d x+\lambda \int_{\Omega} f(x, u) v d x \quad$ are all available to $\forall v \in H_{0}^{s}(\Omega)$. First, let's consider the positive solution of the following questions (1):

$$
f_{+}(x, u)=\left\{\begin{array}{r}
f(x, u) ; u>0 \\
0: u \leq 0
\end{array}\right.
$$

$F_{+}(x, u)=\int_{0}^{u} f_{+}(x, t) d t$, then taking the following problems into account:

$$
\left\{\begin{array}{r}
(-\Delta)^{s} u=\lambda a(x)|u|^{r-2} u+\lambda f^{+}(x, u) ; x \in \Omega \\
u=0 ; x \in \partial \Omega
\end{array}\right.
$$

The corresponding energy functional $I_{\lambda}^{+}(\mathrm{u}): \mathrm{H}_{0}^{s}(\Omega) \rightarrow \mathrm{R}$ is as follow:

$$
I_{\lambda}^{+}(u)=\frac{1}{2} \int_{\Omega}\left|(-\mathrm{V})^{\frac{s}{2}}\right| \mathrm{dx}-\frac{\lambda}{r} \int_{\Omega} a(x)\left(u^{+}\right)^{r} d x-\lambda \int_{\Omega} F_{+}(x, u) d x .
$$

Where $u \in H_{0}^{s}(\Omega)$.

Apparently, $I_{\lambda}^{+} \in C^{1}\left(\mathrm{H}_{0}^{s}(\Omega), \mathrm{R}\right), u$ is the critical point of $I_{\lambda}^{+}$, and $u$ is the solution of (2). Furthermore, according to the principle of the maximum value, $u>0$ in $\Omega$. Therefore, $u$ is also the solution of (1).

Similarly, definite:

$$
\begin{gathered}
f_{-}(x, u)=\left\{\begin{array}{r}
f(x, u) ; \mathrm{u} \leq 0 \\
0: u>0
\end{array}\right. \\
F_{-}(x, u)=\int_{0}^{u} f_{-}(x, t) d t,
\end{gathered}
$$

$I_{\lambda}^{-}(u)=\frac{1}{2} \int_{\Omega}\left|(-V)^{\frac{s}{2}} u\right| \mathrm{dx}-\frac{\lambda}{r} \int_{\Omega} a(x)\left(u^{-}\right)^{r} d x-\lambda \int_{\Omega} F_{-}(x, u) d x$

$I_{\lambda}^{-} \in C^{1}\left(\mathrm{H}_{0}^{s}(\Omega), \mathrm{R}\right), u$ is the critical point of $I_{\lambda}^{-}$. According to the principle of the maximum value, $u<0$ in $\Omega$. So, $u$ is also the solution of (1).

\section{Proof of Main Results}

First, from $\left(H_{1}\right)-\left(H_{3}\right), \quad I_{\lambda}^{+}$and $I_{\lambda}^{-}$are mountain road geometry.

Lemma 1. If hypotheses $\left(H_{1}\right)-\left(H_{2}\right)$ is available, for $\forall \lambda>0$, there exists $\rho, R>0$. Thus, if $\|u\|=\rho, I_{\lambda}^{ \pm} \geq R$.

Proof. By only proving the situation of $I_{\lambda}^{+}$, we can prove the situation of $I_{\lambda}^{-}$by the same way. Get $\alpha \in\left(2, \frac{2 N}{N-2 s}\right)$, from $\left(H_{1}\right)$ and $\left(H_{2}\right)$, which implies there is $C_{\varepsilon}>0$ for $\forall \varepsilon>0$,

$$
F_{+}(x, u) \leq \frac{\varepsilon}{2} u^{2}+C_{\varepsilon} u^{\alpha} \quad \forall x \in \Omega, \forall u>0
$$

According to (4), Poincare' in-equation, Sobolve inequation and Holder in-equation, we have

$$
\begin{aligned}
I_{\lambda}^{+}(u) & \geq \frac{1}{2}\|u\|^{2}-\frac{\lambda}{r}\|a(x)\|_{L^{2}}\|u\|_{L^{2 r}}^{r}-\frac{\lambda \varepsilon}{2} \int_{\Omega}|u|^{2} d x-\lambda C_{\varepsilon} \int_{\Omega}|u|^{\alpha} d x \\
& \geq \frac{1}{2}\|u\|^{2}-C_{r}\|a(x)\|\|u\|^{2}-\frac{\lambda \varepsilon}{2}\|u\|^{2}-C_{\varepsilon}\|u\|^{2} \\
& =\left(\frac{1}{2}-\frac{\lambda}{2} \varepsilon-C_{r}\right)\|u\|^{2}-C_{\varepsilon}\|u\|^{2}
\end{aligned}
$$

Where $C_{s}>0$, taking any small value of $\varepsilon>0$, make sure $\frac{1}{2}-\frac{\lambda}{2} \varepsilon-C_{r} \geq \frac{1}{4}$. Select enough small $\|u\|=\rho>0$, we can find $R>0$, so if $\|u\|=\rho>0$, we have $I_{\lambda}^{+}(u) \geq R$.

Lemma 2. If hypotheses $\left(H_{3}\right)$ is available, $I_{\lambda}^{-}$and $I_{\lambda}^{+}$are no lower bound for $\forall \lambda>0$.

Proof. From $\left(H_{3}\right), \lim _{t \rightarrow+\infty} \inf \frac{1}{t^{2}} \int_{\Omega} F_{+}\left(x, t y(x) d x \geq \frac{1}{2 \varepsilon}\right.$ for $\varepsilon>0$.

Since $\varepsilon$ is arbitrary, so $\lim _{t \rightarrow+\infty} \frac{1}{t^{2}} \int_{\Omega} F_{+}(x, t y(x) d x \rightarrow+\infty$.

We have

$\frac{I_{\lambda}^{+}(t y)}{t^{2}}=\frac{1}{2}\|y\|^{2}-\frac{\lambda t^{r-2}}{r} \int_{\Omega} a(x) y^{r} d x-\frac{\lambda}{t^{2}} \int_{\Omega} F_{+}(x, t y) d x$.

Because of $r>2$, so we have

$$
\lim _{t \rightarrow+\infty} t^{r-2}=+\infty, \lim _{t \rightarrow+\infty} \frac{1}{t^{2}} \int_{\Omega} F_{+}(x, t y) d x=+\infty .
$$




$$
\text { So } \lim _{t \rightarrow+\infty} \frac{I_{\lambda}^{+}(t y)}{t^{2}}=-\infty \cdot I_{\lambda}^{+}(t y) \rightarrow-\infty \text { as } t \rightarrow+\infty \text {. }
$$

The following is to prove that each of the palais-Smale sequences is relatively tight.

Lemma 3. If hypotheses $\left(H_{2}\right)-\left(H_{4}\right)$ is available, every palais-Smale sequence of $I_{\lambda}^{ \pm}$contains a convergent subsequence for all $\lambda>0$.

Proof. It is just to prove the situation of $I_{\lambda}^{+}$is by the same way of $I_{\lambda}^{-}$. Since $\Omega$ is bounded and $\left(H_{2}\right)$ is available, so if $\left\{u_{n}\right\}$ is bounded in $H_{0}^{s}(\Omega)$, we can get strong convergence sequence by Sobolev embedded theorem So it is just to proof that $\left\{u_{n}\right\}$ is bounded in $H_{0}^{s}(\Omega)$.

Supposing $\left\{u_{n}\right\} \subset H_{0}^{s}(\Omega)$ is the palais-Smale sequence of $I_{\lambda}^{+}$, i.e. $I_{\lambda}^{+}\left(u_{n}\right) \rightarrow C_{\lambda},\left(I_{\lambda}^{+}\right)^{\prime}\left(u_{n}\right) \rightarrow 0$, at $n \rightarrow+\infty$, it is suggested to adopt the proof by contradiction. We suppose that $\left\|u_{n}\right\| \rightarrow+\infty$ as $n \rightarrow+\infty$. If setting $w_{n}=\frac{u_{n}}{\left\|u_{n}\right\|}$ and $\left\|w_{n}\right\|=1$. We may assume that for some $w \in H_{0}^{s}(\Omega)$, it makes $w_{n}$ weak convergence of $w$ in $H_{0}^{s}(\Omega), w_{n} \rightarrow w$ and in $L^{2}(\Omega)$, furthermore $w_{n}(x) \rightarrow w(x)$, a.e. $x \in \Omega$. From $\left(H_{3}\right)$, by combining Fatou lemma, we have $w(x) \equiv 0$, a.e. $x \in \Omega$. Set $t_{n} \in[0,1]$ such that $I_{\lambda}^{+}\left(\mathrm{t}_{n} u_{n}\right)=\max _{t \in[0,1]} I_{\lambda}^{+}\left(\mathrm{t} u_{n}\right)$, which implies that: $\left.\frac{d}{d t} I_{\lambda}^{+}\left(t u_{n}\right)\right|_{t=t_{n}}=t_{n}\left\|u_{n}\right\|^{2}-\lambda t_{n}^{r-1} \int_{\Omega} a(x) u_{n}^{r} d x-\lambda \int_{\Omega} f_{+}\left(x, t_{n} u_{n}\right) d x=0$.

Since

$\left(I_{\lambda}^{+}\right)^{\prime}\left(\mathrm{t}_{n} u_{n}\right) \cdot\left(\mathrm{t}_{n} u_{n}\right)=t_{n}^{2}\left\|u_{n}\right\|^{2}-\lambda t_{n}^{r} \int_{\Omega} a(x) u_{n}^{r} d x-\lambda \int_{\Omega} f_{+}\left(x, t_{n} u_{n}\right) t_{n} u_{n} d x \cdot$

We have $\left(I_{\lambda}^{+}\right)^{\prime}\left(\mathrm{t}_{n} u_{n}\right) \cdot\left(\mathrm{t}_{n} u_{n}\right)=\left.t_{n} \frac{d}{d t} I_{\lambda}^{+}\left(t u_{n}\right)\right|_{t=t_{n}}=0$.

From $\left(H_{4}\right)$, we have

$$
\begin{aligned}
& 2 I_{\lambda}^{+}\left(\mathrm{tu}_{n}\right) \leq 2 I_{\lambda}^{+}\left(\mathrm{t}_{n} \mathrm{u}_{n}\right)-\left(I_{\lambda}^{+}\right)^{\prime}\left(\mathrm{t}_{n} \mathrm{u}_{n}\right)\left(\mathrm{t}_{n} \mathrm{u}_{n}\right) \\
& =\lambda \int_{\Omega}\left(t_{n} u_{n} f_{+}\left(x, t_{n} u_{n}\right)-2 F_{+}\left(x, t_{n} u_{n}\right)\right) \mathrm{d} x+\lambda\left(1-\frac{2}{r}\right) t_{n}^{r} \int_{\Omega} a(x) u_{n}^{r} d x \\
& \leq \lambda \int_{\Omega}\left(u_{n} f_{+}\left(x, u_{n}\right)-2 F_{+}\left(x, u_{n}\right)+\mathrm{C}_{*}\right) \mathrm{d} x+\lambda\left(1-\frac{2}{r}\right) \int_{\Omega} a(x) u_{n}^{r} d x \quad \mathrm{O} \\
& =2 I_{\lambda}^{+}\left(\mathrm{u}_{n}\right)-\left(I_{\lambda}^{+}\right)^{\prime}\left(\mathrm{u}_{n}\right)\left(\mathrm{u}_{n}\right)+\lambda|\Omega| C_{*} \\
& =2 C_{\lambda}+\lambda|\Omega| C_{*} \quad \mathrm{n} \text { the other side, } \forall R_{0}>0 \\
& 2 I_{\lambda}^{+}\left(\mathrm{R}_{0} w_{n}\right)=\mathrm{R}_{0}{ }^{2}-\frac{2 \lambda}{r} \int_{\Omega} a(x)\left(\mathrm{R}_{0} w_{n}\right)^{r} d x-2 \lambda \int_{\Omega} F_{+}\left(x, \mathrm{R}_{0} w_{n}\right) d x \\
& =\mathrm{R}_{0}{ }^{2}+o(1)
\end{aligned}
$$

\section{The proof of the theorem 1.}

From $\left(H_{1}\right)$ we know $I_{\lambda}^{ \pm}(0)=0$. By lemma 2, there is $e \in H_{0}^{s}(\Omega),\|e\|>\rho$ satisfies $I_{\lambda}^{ \pm}(e)<0$.Besides, there are $\rho$ and $\mathrm{R}>0$, so we have $\left.I_{\lambda}^{ \pm}(u)\right|_{\partial B_{\rho}} \geq R$.

Define

$$
c_{\lambda}^{ \pm}=\inf _{\gamma \in \Gamma} \max _{t \in[0,1]} I_{\lambda}^{ \pm}(\gamma(t)),
$$

Where

$$
\Gamma=\left\{\gamma:[0,1] \rightarrow H_{0}^{s}(\Omega): \gamma \text { continuous, } \gamma(0)=0, \gamma(1)=e\right\} \text {. }
$$

By lemma 3, we have $I_{\lambda}^{ \pm}$satisfying the Palais-Smale condition. By mountain pass theorem, we know that $c_{\lambda}^{+}$is the critical value of $I_{\lambda}^{+}$and there are at least a nontrivial critical point $u_{\lambda}^{+} \in H_{0}^{s}(\Omega)$ satisfying $I_{\lambda}^{+}\left(u_{\lambda}^{+}\right)=c_{\lambda}^{+}$.Clearly, $u_{\lambda}^{+} \geq 0$. By the strong maximum principle, we have $u_{\lambda}^{+}>0$, so $u_{\lambda}^{+}$is the positive solution of (1). In the say way, there are at least a negative solution $u_{\lambda}^{-} \in H_{0}^{s}(\Omega)$. As a result, there are at least a positive solution and a negative solution on problem (1).

\section{REFERENCES}

[1] Servadei R and Valdinoci E, Mountain Pass Solutions for Nonlocal Elliptic Operators. J. Math .Anal. Appl.,2012,389(2):887-898.

[2] Zhang Shifeng and Yang Ganshan(PRC-YNN-IM), Pairs of positive solutions for a class of p-laplacian equations. Math. Sci. Res. J.15(2011), no.1,12-19.

[3] Miyagaki O H and Souto M A S, Superlinear problems Without the Ambrosetti and Rabinowitz Growth Condition. J. Differential Equations, 2008, 245(12):3628-3638

[4] Liu Shibo, On Superlinear Problems Without the Ambrosetti and Rabinowitz Growth Condition. Nonlinear Anal., 2010, 73(3):788-795.

[5] Bartsch T, Liu Zhao-li and Weth T, Nodal Solutions of a p-laplacian Equation. Pro. London Math. Soc., 2005,91(1):129-152.

[6] Valdinoci E, From the Long Jump Random Walk to the Fractional Laplacian. Bol. Soc. Esp. Mat. Apl. SMA., 2009, 49(1):33-44.

[7] Applebaum D, Levy Processes-from Probability to Finance and Quantum Groups. Notices Amer. Math . Soc., 2004,51(11):1336-1347.

[8] Wei Yuanhong, Chang Xiaojun and Lu Yue, Superlinear Fourth-order Elliptic Problem Without Ambrosetti - Rabinowitz Growth Condition. Comm. Math. Reach, 2013,29(1):23-31.

[9] Bradle C, Colorado E and DE Pablo A. A Concave-convex Elliptic Problem Involving the Fractional Laplacian. Proc. Roy. Soc. Edinburgh Sect. A, 2013,143(1):39-71. 